\title{
Total Ankle Replacement: A Review
}

\author{
${ }^{1}$ Jariwala, A., ${ }^{2}$ S. Sripada and ${ }^{2}$ A. Pillai \\ ${ }^{1}$ Department of Orthopaedic and Trauma Surgery TORT Centre, \\ Clinical Lecturer University, Ninewells Hospital and Medical School Dundee, Scotland \\ ${ }^{2}$ Department of Orthopaedic and Trauma Surgery Ward 18-19, \\ Level 5, Ninewells Hospital Dundee, Scotland
}

Received 2012-05-14, Revised 2013-01-16; Accepted 2013-01-17

\begin{abstract}
Despite the initial failures of ankle arthroplasty, the newer generations of total ankle replacement have shown good medium term results. Reasons stated for this change include a better overall understanding of ankle biomechanics; lessons learnt from hip and knee arthroplasty, improved ankle implant designs that better replicate the human anatomy and finally in the development of foot and ankle surgery as a subspeciality with committed surgeons. Literature supports the use of total ankle replacement for advanced ankle arthritis in elderly patients with moderately active lifestyle but recommends counselling patients appropriately regarding reasonable expectations and complications. This review looks at the development of total ankle replacement, present indications; the surgical technique, the results from various centres and indicates the present position regarding the management of ankle arthritis.
\end{abstract}

Keywords: Total Ankle Replacements (TAR), Previously Mentioned First Generation, Treatment Modality, Neuropathic Joints or Post-Infectiona, Biomechanical Reasons

\section{INTRODUCTION}

Improved understanding of ankle biomechanics over the past few decades has revolutionised the development and design of total ankle replacement implants. Initial attempts at Total Ankle Replacements (TAR) were fraught with problems. Poor understanding of ankle biomechanics, inferior metallurgy and predominant focus of the industry towards the development of hip and knee arthroplasty were just a few of the factors delaying the emergence of total ankle replacement as a treatment modality for ankle arthritis.

Unlike hip and knee, primary osteoarthritis rarely affects the ankle joint (Cushnaghan and Dieppe, 1991). The cause for this discrepancy is unclear although some anatomical and biomechanical reasons have been proposed. The ankle joint has stiffer cartilage compared to other joints, behaves like a rolling joint and is a highly congruent joint thereby creating uniform stress distribution and reducing wear (Treppo et al., 2000; Shepherd and Seedhom, 1999; Kempson, 1991; Ramsey and Hamilton, 1976). Being a highly congruent joint predisposes the ankle joint to be intolerant of any joint incongruencies or abnormal motion. Hence secondary osteoarthritis is much more common in the ankle joint than primary osteoarthritis. The main causes of secondary osteoarthritis are post-traumatic or in patients having ankle instability. In addition, ankle arthritis may present in conditions such as inflammatory arthritis (for example rheumatoid arthritis), neuropathic joints or post-infection.

Proponents of TAR outline its benefits citing increased ankle mobility, relief from arthritic pain, improved gait and function and possibly reduced incidence of progression of arthritis to the sub-talar joint and transverse tarsal joints in comparison to ankle arthrodesis (Knecht et al., 2004; Wood and Deakin, 2003). While opponents allude to increased postoperative complications, lack of long-term survivorship data and problems with salvage of failed ankle replacement as concerns regarding TAR.

This review investigates the history and development of TAR and indicates its present position regarding the management of ankle arthritis.

Corresponding Author: Jariwala, A., Department of Orthopaedic and Trauma Surgery TORT Centre, TORT Centre, Clinical Lecturer University, Ninewells Hospital and Medical School Dundee, Scotland 


\subsection{Evolution of TAR}

First generation TAR was introduced in the early 1970. There were reports of implanting the hip prosthesis in the tibia and the acetabular plastic cup in the calcaneus as an initial attempt to replace the ankle joint (Vickerstaff et al., 2007). It was no surprise that they recommended fusing the ankle due to poor surgical results.

The first generation designs were predominantly twopart designs incorporating a polyethylene concave surface and metal convex surface. These were secured with cement and were either constrained or unconstrained. The constrained designs suffered from problems of stiffness and early loosening while the unconstrained designs showed problems of instability leading to failure. Survivorship at 5.5 years was approximately 20\% (Bolton-Maggs et al., 1985). Major complications were noted including wound breakdown, infections, talar collapse and loosening of components leading to a tendency for surgeons to promote ankle fusion over ankle replacement.

As previously mentioned first generation TAR were primarily two-part systems possibly due to the fact that the ankle joint was thought to be a simple hinge with dorsiflexion and plantarflexion movements. It was an improved understanding of ankle motion that highlighted that the ankle biomechanics was more complex than previously believed with contributions from the subtalar joint and rotation and sliding motion in addition, to predominant dorsiflexion and plantarflexion movements.

Experiences from clinicians using first generation designs such as New Jersey Cylindrical Replacement (NJCR) and Irvine ankle arthroplasty designs further aided the improvement in TAR design. They recommended the use of congruent designs; less constraining implants allowing rotation in addition to sagittal plane movements. In addition, they advocated the use of cementless designs as cemented designs required larger bone cuts thus leading to loosening and thereby failure (Hvid et al., 1985).

Second generation TAR implants were designed based upon the experiences resulting from the failures of first generation implants. The implants were modified to replicate the ankle anatomy and biomechanics. They were designed to be used cementless and were semiconstrained. This required less bony resection and thus greater bone conservation. They were either two or three part designs which aimed to replicate the normal ankle motion and biomechanics.

The two-part designs such as the Agility ankle are semi-constrained devices containing a large tibial component and a smaller talar component into which the modular polyethylene inserts lock (Fig. 1). In addition, the Agility ankle required the concomitant fusion of lower tibio-fibular syndesmosis. The results of two-part Agility ankles were promising with up to $80 \%$ survival at 4.8 years (Pyevich et al., 1998).

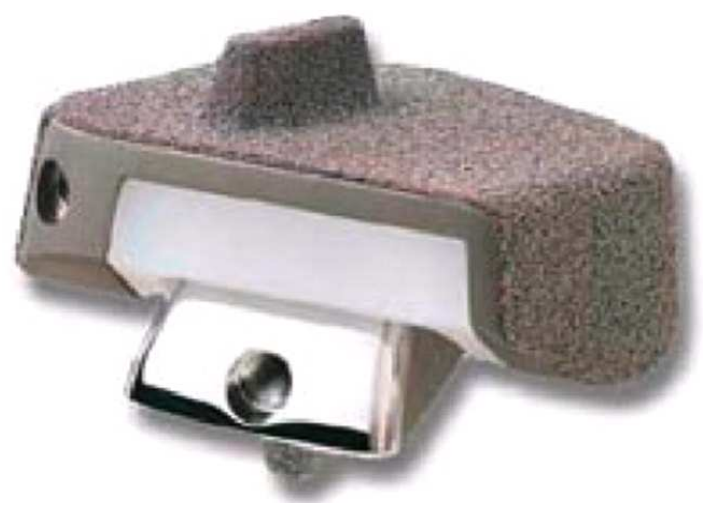

Fig. 1. Two-component total ankle replacement: Agility ankle

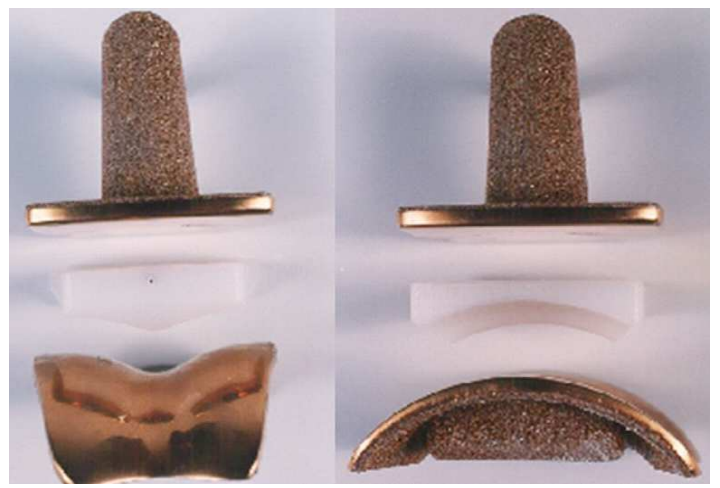

Fig. 2. Three-component total ankle replacement: BuechelPappas ankle

The three component design comprises of two metal components; one for the under surface of the tibia and the other for the dome of the talus. Both metal components are porous coated for osseo-integration. A plastic bearing sits between the two metal trays (Fig. 2). The three component design allows flexion-extension, a certain amount of rotation and sliding therefore replicating the majority of normal ankle movements. Most designs currently in use are three component designs such as the Buechel-Pappas and STAR ankles. The inventor series noted the survival of the STAR ankle to be $92 \%$ (Wood and Deakin, 2003) with the main problems being wound complications and per-operative fractures of the malleoli.

With improving results from ankle replacement newer designs have emerged such as the Salto ankle, Hintegra ankle and Mobility TAR with nearly all using the basic three-part design with some modifications.

\subsection{Indications and Contraindications}

An optimal patient for TAR is one who is older than 50 years of age, has advanced ankle arthritis, is not obese and who has low physical expectations. 

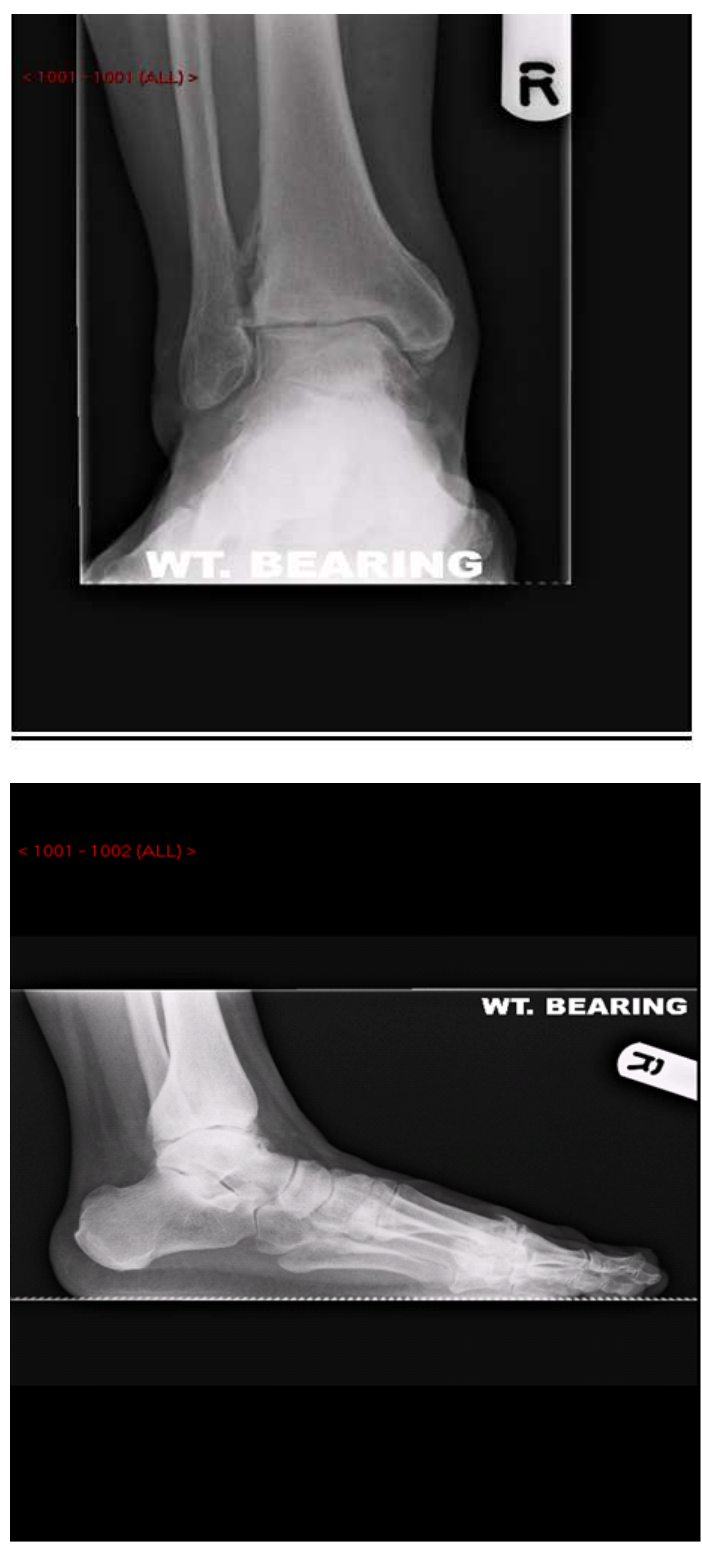

Fig. 3. Ankle radiographs demonstrate signs of arthritis like reduced joint space, sclerosis and osteophytes

Younger patients place higher demands on the prosthesis and as a result can cause failure by loosening. It is important to note the arthritis involvement of neighbouring joints, although this is not a contraindication.

Absolute contraindications for TAR are active infection, poor soft tissue envelope, severe deformity or instability, neuropathic joint, avascularity of the talus and peripheral vascular disease. Ankle replacement may be avoided in patients with substantial activity levels or those involved in high-impact sports.

\subsection{Pre-Operative Planning}

Work up for TAR includes taking a thorough history and examination investigating the aetiology of ankle arthritis, any disability from the arthritis, associated co-morbidities and previous operative and non-operative treatment. Appropriate weight-bearing radiographs are required to assess the stage of arthritis in the ankle, instability, deformity and any involvement of other joints (Fig. 3). Long leg radiographs are helpful in planning TAR in patients with deformity. Intraoperative imaging is essential.

\subsection{TAR Surgery}

TAR is generally carried out with the patient under spinal anaesthesia or regional blocks in a supine position. A tourniquet is used to allow a bloodless surgical field. Anterior approach to the ankle protecting the neurovascular structures and the tendons is made. The ankle joint is exposed and osteophytes, inflammatory synovium and joint capsule are excised. Medial or lateral releases are performed to correct the soft tissue alignment as appropriate.

An alignment guide is placed on the tibia and the tibial cut made. Thereafter the talar cut is made and the trial is undertaken. The trial assembly is verified for correct positioning and orientation. Final implants are seated in and plastic spacer inserted (Fig. 4). Any ligament imbalance, deformity, Achilles tendon tightness and neighbouring joint involvement are addressed appropriately. Finally, a thorough check of alignment, position and range of motion is done before tourniquet release. Closure is performed in layers and bulky pressure dressing is administered. Some surgeons tend to immobilise the ankle in a below knee plaster slab for the first few weeks after surgery. Thereafter the patient can be immobilised in a cast or walking boot depending on local protocols.

\subsection{Post-Operative Management}

General TAR ankle movements are allowed six weeks after surgery. Radiographs are taken to ensure proper alignment of implants when weight bearing (Fig. 5). Postoperative regimes for TAR with reconstructive procedures such as ligament reconstruction or osteotomies for deformity are dictated by any additional procedures. Follow-up after the acute period is variable but most foot and ankle units tend to follow TAR analogous to other arthroplasties. 


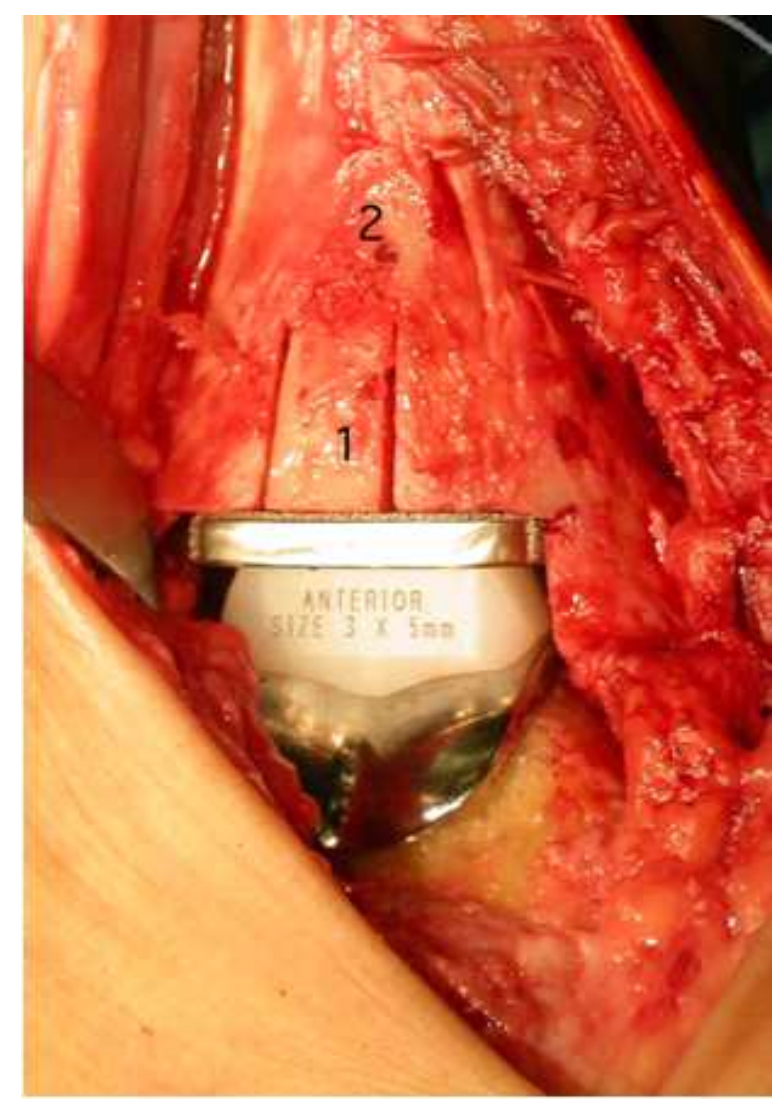

Fig. 4. Per-operative final position of the implants with plastic spacer in between

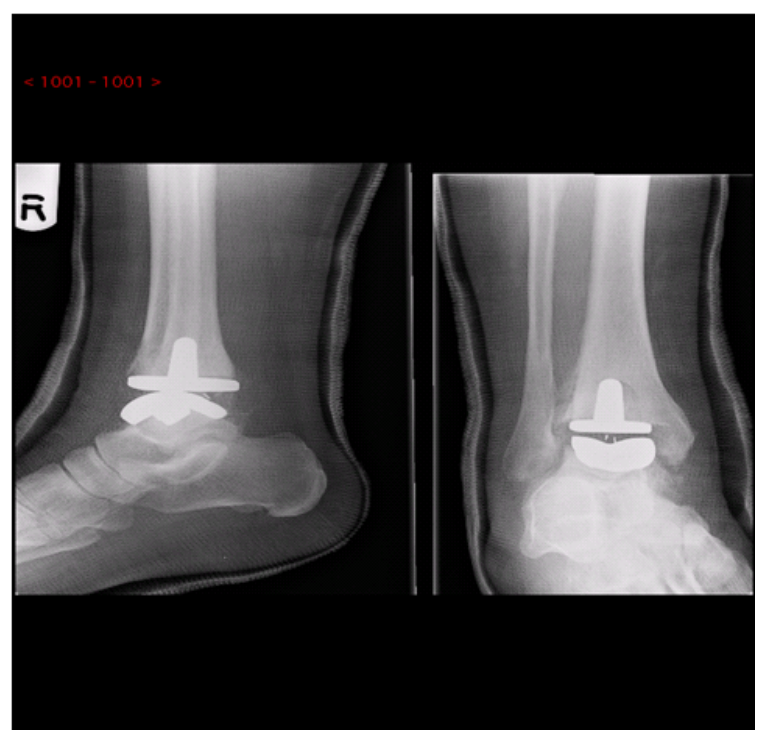

Fig. 5. Radiographs indicating proper alignment of implants

\subsection{Complications}

Common complications arising from TAR include wound problems and infection, hence meticulous attention to soft tissue dissection is critical. Malalignment and improper implant placement are common technical errors and lead to asymmetrical loading and ultimately failure. Associated ligament imbalance, deformity and neighbouring joint involvement if not corrected during surgery may lead to poor outcome. Loosening of implants in very active individuals or obese patients is a noted complication. Salvage of failed ankle replacement, especially after infection, is a difficult situation and best avoided. Arthrodesis requiring bone grafts may be the only available option. Currently there are no established revision options.

\section{RESULTS}

Results of the present generation of TAR are promising. Various studies have noted medium to longterm survival rates between 75-95\% (Knecht et al., 2004; Wood and Deakin, 2003). Most of these results comprise of series from inventor or specialist centres. Gougoulias et al. (2010) in their review of 1105 different TAR noted that ankle function improved after TAR. They noted trauma and rheumatoid arthritis as the most common indications for TAR. Looking critically through the studies from 1990-2005 the authors of this systematic review noted an approximate $77 \%$ survival rate for TAR with a $10 \%$ failure rate (Gougoulias et al., 2010). Recent reviews with newer implants have noted improved survival rates for medium term follow-up ( $90 \%$ at 5 years). The Swedish joint registry noted the effect of learning curve with improvement in survival rates after surgeons had performed 30 TARs (Henricson et al., 2007).

Most studies find that there is an improvement in pain post-operatively $(60-80 \%)$ and improved patient satisfaction. There is limited improvement in the ankle range of motion (0-14 degrees) and both residual pain and complications are common (Gougoulias et al., 2010).

Randomised trials comparing TAR to ankle fusion are limited and report better function, improved gait characteristics and comparable pain relief with TAR as compared to ankle arthrodesis (Dyrby et al., 2004; Haddad et al., 2007). In addition, a reduced need for subtalar fusion after TAR in comparison to ankle fusion was noted.

\section{CONCLUSION}

Primary ankle arthritis although not as common as hip or knee arthritis can still be equally disabling. Improvements in TAR implant designs and 
instrumentation, better understanding of biomechanics and dedicated surgeons have placed TAR on an equal pedestal with ankle fusion for advanced ankle arthritis. Literature supports the use of TAR for advanced ankle arthritis in patients ages over 50 years with moderately active lifestyle but recommends counselling patients appropriately regarding reasonable expectations, increased complications and lack of long-term results for newer TAR designs.

\section{ACKNOWLEDGEMENT}

We wish to thank Mr Ian Christie for assisting with the editing of the manuscript and the illustrations. We have obtained the implant pictures from the Internet and also from the specific company websites.

\section{REFERENCES}

Bolton-Maggs, B.G., R.A. Sudlow and M.A. Freeman, 1985. Total ankle arthroplasty. A long-term review of the london hospital experience. J. Bone Joint Surg. Br., 67: 785-790. PMID: 4055882

Cushnaghan, J. and P. Dieppe, 1991. Study of 500 patients with limb joint osteoarthritis. I. Analysis by age, sex, and distribution of symptomatic joint site. Ann. Rheum Dis., 50: 8-13. PMID: 1994877

Dyrby, C., L.B. Chou, T.P. Andriacchi and R.A. Mann, 2004. Functional evaluation of the scandinavian total ankle replacement. Foot Ankle Int., 25: 377-381. PMID: 15215020

Gougoulias, N., A. Khanna and N. Maffulli, 2010. How successful are current ankle replacements. Clin. Orthop. Relat. Res., 468: 199-208. DOI: 10.1007/s1 1999-009-0987-3

Haddad, S.L., J.C. Coetzee, R. Estok, K. Fahrbach and D. Banel et al., 2007. Intermediate and long-term outcomes of total ankle arthroplasty and ankle arthrodesis. A systematic review of the literature. J. Bone Joint Surg Am., 89: 1899-1905. PMID: 17768184
Henricson, A., A. Skoog and A. Carlsson, 2007. The swedish ankle arthroplasty register: An analysis of 531 arthroplasties between 1993 and 2005. Acta Orthop., 78: 569-574. PMID: 17966014

Hvid, I., O. Rasmussen, N.C. Jensen and S. Nielsen, 1985. Trabecular bone strength profiles at the ankle joint. Clin. Orthop, 199: 306-312. PMID: 4042494

Kempson, G.E., 1991. Age-related changes in the tensile properties of human articular cartilage: A comparative study between the femoral head of the hip joint and the talus of the ankle joint. Biochim. Biophys. Acta, 1075: 223-2. PMID: 1954224

Knecht, S.I., M. Estin, J.J. Callaghan, M.B. Zimmerman and K.J. Alliman, 2004. The agility total ankle arthroplasty. Seven to sixteen-year follow-up. J. Bone Joint Surg, Am., 86-A: 1161-1171. PMID: 15173288

Pyevich, M.T., C.L. Saltzman, J.J. Callaghan and F.G. Alvine, 1998. Total ankle arthroplasty: A unique design. two to twelve-year follow-up. J. Bone Joint Surg, Am., 80: 1410-1420.

Ramsey, P.L. and W. Hamilton, 1976. Changes in tibiotalar area of contact caused by lateral talar shift. J. Bone Joint Surg. Am., 58: 356-357. PMID: 1262367

Shepherd, D.E. and B.B. Seedhom, 1999. Thickness of human articular cartilage in joints of the lower limb. Ann. Rheum Dis., 58: 27-34. PMID: 10343537

Treppo, S., H. Koepp, E.C. Quan, A.A. Cole and K.E. Kuettner et al., 2000. Comparison of biomechanical and biochemical properties of cartilage from human knee and ankle pairs. J. Orthop. Res., 18: 739-748. PMID: 11117295

Vickerstaff, J.A. W.A. Miles and J.L. Cunningham, 2007. A brief history of total ankle replacement and a review of the current status. Med. Eng. Phys., 29: 1056-1064. PMID: 17300976

Wood, P.L. and S. Deakin, 2003. Total ankle replacement. The results in 200 ankles. J. Bone Joint Surg. Br., 85: 334-341. PMID: 12729104 